\title{
Correlation Between Anti Mullerian Hormone Level and Antral Follicle Count Using Three-Dimensional Ultrasound in Cases of Recurrent Spontaneous Abortion
}

\author{
Khaled Zakaria El-Sheikha ${ }^{\mathbf{1}}$, Abd El Monsef Abd Elhamid Sedek ${ }^{\mathbf{1}}$, \\ Ibrahim Hassan Mohammed ${ }^{2}$, Ahmed Mohamed Hussein Hafez ${ }^{1}$ \\ ${ }^{1}$ Obstetrics \& Gynecology Department and ${ }^{2}$ Biochemistry Department, Faculty of Medicine, \\ Al-Azhar University, Cairo, Egypt \\ * Corresponding author: Ahmed Mohamed Hussein Hafez, E-mail: drahmedhussin8@ gmail.com
}

\begin{abstract}
Background: abortion is one of the most common complications of pregnancy, occurring in 10-15\% of pregnant women, whiles recurrent spontaneous abortion, rate of $2-5 \%$, is defined as $\geq 3$ spontaneous abortions.
\end{abstract}

Aim of the Work: was to try to find relationship between serum AMH concentration which represent ovarian reserve, Antral Follicular count and any pelvic Structural abnormality using 3D ultrasound in assessment cases with spontaneous recurrent abortion.

Patients and Methods: A multicenter, randomized trial included 100 women who were divided into 2 groups; Group 1, included 50 patients with $\geq 3$ recurrent abortions and Group 2, included 50 parous women with regular menstrual cycles and having one or more living child without any history of pregnancy loss or pelvic structural abnormality. Every subject was subjected to assessing hormonal profile including anti-müllerian hormone (AMH) and Transvaginal ultrasonography.

Results: AMH was found to have negative correlation with age, number of abortions, FSH and LH.

Conclusion: It could be concluded that Anti-Müllerian hormone is the best indicator of ovarian reserve with a high sensitivity and specificity, so ovarian reserve of women with recurrent spontaneous abortions is not defective.

Keywords: Anti Mullerian Hormone Level, Antral Follicle Count, Three-Dimensional Ultrasound, Recurrent Spontaneous Abortion

\section{INTRODUCTION}

Abortion is one of the most common complications of pregnancy, occurring in 10 $15 \%$ of pregnant women. It is defined as pregnancy that fails to progress resulting in death of the fetus before age of fetal viability $\left(20^{\text {th }}\right.$ week (weight of $\leq 500 \mathrm{~g}$ ) in developed countries and $28^{\text {th }}$ week (weight of $\leq 1 \mathrm{~kg}$ ) in developing countries) ${ }^{(1)}$.

Recurrent abortion, rate of $2-5 \%$, is defined as $\geq 3$ spontaneous abortions. In these women, it is necessary to conduct a comprehensive evaluation so that a plan of care can be outlined ${ }^{(1)}$.

On January 2008 has defined recurrent pregnancy loss by two or more failed pregnancies. When the cause is unknown, each pregnancy loss merits careful review to determine whether specific evaluation may be appropriate. After three or more losses, a thorough evaluation is warranted. For purposes of determining when evaluation and treatment for infertility or for recurrent pregnancy loss is appropriate, pregnancy is defined as a clinical pregnancy documented by ultrasonography or histopathologic examination ${ }^{(2)}$.

Anti müllerian hormone (AMH) is a dimeric glycoprotein made up of two monomers attached to each other by disulfide bonds. The 72-kd molecule belongs to the transforming growth Factor-B superfamily, which acts on tissue growth and differentiation. Sertoli cells in the male produce $\mathrm{AMH}$, which induces the degeneration of the müllerian ducts and provides the normal formation of the male genital system. Sertoli cells' secretion of AMH continues for a lifetime, but the significance of $\mathrm{AMH}$ in adult male is not known. In females, the granulosa cells of the ovary express AMH postnatally. Serum AMH levels are lower in 
females compared with males. After puberty and onset of menstrual cycles, serum AMH level decreases progressively until it becomes undetectable at around menopause ${ }^{(3)}$.

Anti-Müllerian hormone can first be detected in human fetal ovary at 36th week in columnar granulosa cells of maturing primary follicles. AMH expression persists in these follicles and is maximally expressed in granulosa cells of preantral and small antral follicles (up to $6 \mathrm{~mm}$ ). At larger antral follicle stage $(>8 \mathrm{~mm})$, its expression diminishes and ultimately becomes undetectable once FSH dependent follicular growth has been initiated. No $\mathrm{AMH}$ expression is detected in atretic follicles ${ }^{(4)}$.

Secreted from preantral and early antral follicles, $\mathrm{AMH}$ regulates ovarian activity and follicular steroidogenesis. Animal studies have revealed that not only does AMH decrease aromatase activity of FSH-stimulated granulosa cells, but it also decreases the number of luteinizing hormone (LH) receptors, and regulates testosterone production in theca cells ${ }^{(5)}$.

The regulation of oocyte function is through endocrine and paracrine factors. Although the basic hormones causing oocyte growth are $\mathrm{FSH}$ and $\mathrm{LH}$, receptors for gonadotropins could not be found on oocytes. The action of gonadotropins on oocytes is probably through mediators like epithelial growth factor (EGF), vascular endothelial growth factor (VEGF), insulin-like growth factor 2 (IGF2). The intra follicular androgen to estrogen ratio also acts on oocyte function, and AMH plays a major role in the regulation of this ratio ${ }^{(6)}$.

The pattern of expression of AMH strongly indicates that $\mathrm{AMH}$ has an important role in regulating the number of follicles that grow from the primordial pool. Moreover, AMH might regulate selection of the dominant follicle from the FSH-sensitive follicle cohort (4).

Serum AMH levels were more robustly correlated with the number of early antral follicles than $\mathrm{E}_{2}, \mathrm{FSH}$ and $\mathrm{LH}$ on cycle day 3. This suggests that AMH may reflect ovarian follicular status better than the usual hormone markers ${ }^{(7)}$.
On the other hand, ovarian reserve is a term used to describe the functional potential of the ovary and reflects the number and quality of oocytes within it ${ }^{(8)}$.

AMH was the best indicator of ovarian reserve with a high sensitivity and specificity. Levels of AMH would predict the number of oocytes with a positive predictive rate of $96 \%$, although it had little value for predicting pregnancy ${ }^{(6)}$.

The process of follicular recruitment and selection takes place during the follicular phase of the cycle. Hence, events during the follicular phase may affect the pregnancy outcome. However, most studies on recurrent abortion have focused on the luteal phase of menstrual cycle, and there is limited information on the follicular phase and correlated ovarian reserve in women with recurrent abortions, especially cases with unexplained causes ${ }^{(9)}$.

The aim of this work was to try to find relationship between serum AMH concentration which represent ovarian reserve, Antral Follicular count and any pelvic Structural abnormality using 3D ultrasound in assessment cases with spontaneous recurrent abortion.

\section{PATIENTS AND METHODS}

This 2-arm, prospective, open-labeled, multicenter, randomized study included a total of 50 women with $\geq 3$ recurrent abortions and 50 parous women with regular menstrual cycles and having one or more living child without any history of pregnancy loss or pelvic structural abnormality, serving as control. They were attending at Al-Azhar University Hospitals (Al-Hussein \& Bab El-Sharia) in Cairo and at Al-Entag Al-Harby Hospital at Helwan. Approval of the ethical committee and a written informed consent from all the subjects were obtained. This study was conducted between December 2016 and May 2018.

\section{Inclusion criteria are:}

1. Age between 20 and 32 years.

2. History of $\geq 3$ recurrent consecutive spontaneous abortions between 10-16 weeks of gestation for study group.

3. Body mass index between 19 and 29 . 
4. Regular menstrual cycles, no prior pregnancy loss for and proven fertility by having one or more living child for control group.

\section{Exclusion criteria are:}

1. Known cases of decreased ovarian reserve.

2. Anti-phospholipid syndrome and other thrombophilic disorders.

3. Thyroid gland abnormalities.

4. Hyperprolactinemia.

5. Diabetes mellitus.

6. Known genetic disease in either parent.

7. Pelvic structural abnormality (including cervical incompetence).

\section{Every subject was subjected to:}

1. Detailed history to ensure inclusion and exclusion criteria.

2. Physical examination and assessment of body mass index.

3. On day three, withdrawal a venous blood sample to assess serum Hormonal profile: follicle stimulating hormone (FSH), luteinizing hormone (LH), prolactin, thyroid releasing hormone (TSH) and antimüllerian hormone (AMH).

4. Transvaginal ultrasonography: with the application of high-resolution vaginal ultrasound (mindray DC-N3), the problems which were previously encountered during transabdominal scanning, such as obesity, bowel gas, retroverted uterus and inability to permit full bladder distention no longer preclude accurate diagnosis with patient acceptance for the transvaginal scan. So, a management and treatment become easier and faster.

\section{Statistical analysis}

Data were coded and entered using the statistical package SPSS version 25. Data was summarized using mean and standard deviation for normally distributed quantitative variables or median and interquartile range for non-normally distributed quantitative variables and frequencies (number of cases) and relative frequencies (percentages) for categorical variables. Comparisons between groups were done using unpaired $t$ test in normally distributed quantitative variables while nonparametric Mann-Whitney test was used for non-normally distributed quantitative variables. For comparing categorical data, Chi square $(\chi 2)$ test was performed. Exact test was used instead when the expected frequency is less than 5. Correlations between quantitative variables were done using Spearman correlation coefficient. P-values less than 0.05 were considered as statistically significant. $\mathrm{P}$ value > 0.05: insignificant, $\mathrm{P}$ value $<0.05$ : significant, $\mathrm{P}$ value $<0.01$ : highly significant.

\section{RESULTS}

$>$ the demographic characteristics of the participants in this study including the age, body mass index, number of previous abortions and parity are presented in table (1) \& figure (1): -

\begin{tabular}{|l|c|c|c|}
\hline \multicolumn{1}{|c|}{ Participant characteristics } & $\begin{array}{c}\text { (Group 1) (n=50) } \\
\text { Study group }\end{array}$ & $\begin{array}{c}\text { (Group 2) }(\mathbf{n = 5 0}) \\
\text { Control group }\end{array}$ & P value \\
\hline Age at randomization, y & $27.47 \pm 3.20$ & $28.57 \pm 3.48$ & 0.208 \\
\hline Body mass index b & $25.84 \pm 2.75$ & $25.23 \pm 2.91$ & 0.414 \\
\hline Average No. of previous abortions & $4.23 \pm 1.16$ & 0 & ----- \\
\hline Parity (No \%) & $41(82 \%)$ & $0(0 \%)$ & \\
NG & $8(16 \%)$ & $30(60 \%)$ & \\
P1 & $1(2 \%)$ & $11(22 \%)$ & $<0.001$ \\
P2 & $0(0 \%)$ & $9(18 \%)$ & \\
P3 & 0 & \\
\hline
\end{tabular}

Table (1): Shows the demographic characteristics of the participants used in this study 

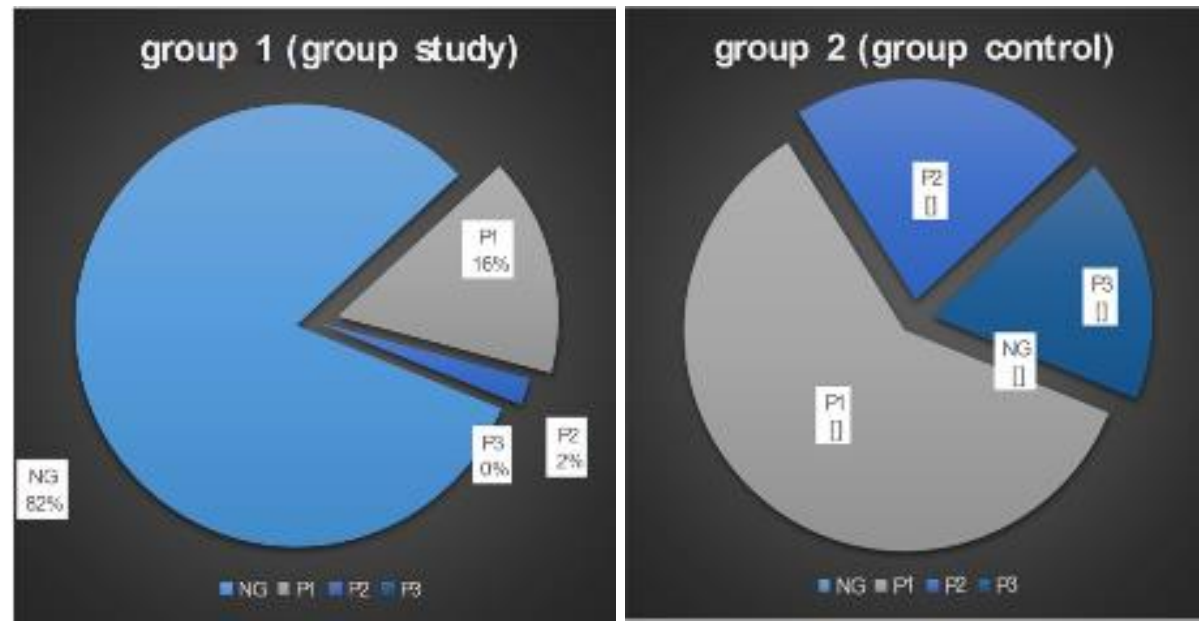

Figure (1): Pie-Chart showing Distribution of Parity among Included Women.

$>$ Statistical comparison shows a highly significant difference between (group 1) and (group 2) as regard prolactin levels as shown in table (2) \& figure (2) and it was due to cases showed significantly higher prolactin levels: -

\begin{tabular}{|c|c|c|c|c|}
\hline & Group 1 $(\mathbf{n = 5 0})$ & Group 2 $(\mathbf{n = 5 0})$ & P value & Sig \\
\hline Prolactin $\mathrm{ng} / \mathrm{ml}$. & $15.66 \pm 2.19$ & $12.33 \pm 2.48$ & $<0.00$ & $\mathrm{~S}$ \\
\hline
\end{tabular}

Table (2): Statistical comparison between patients (group 1) and controls (group 2) regarding prolactin profile.

$>$ Statistical comparison between (group 1) and (group 2) shows positive correlation between $\mathrm{AMH}$ and AFC and shows negative correlation between $\mathrm{AMH}$, age, number of abortions, FSH, LH and E2 as demonstrated in table (3): -

\begin{tabular}{|c|c|c|c|c|c|}
\hline & & \multicolumn{2}{|c|}{$\begin{array}{c}\text { (Group 1) } \\
\text { Study group =15) }\end{array}$} & \multicolumn{2}{|c|}{$\begin{array}{c}\text { (Group 2) } \\
\text { Control group=15) }\end{array}$} \\
\hline & & rho value & P value & rho value & P value \\
\hline \multirow{2}{*}{ Clinical Data: } & Age in years & $-0.51(* *)$ & 0.002 & -0.641 & $<0.0001$ \\
\hline & Number of abortions & $-0.42(*)$ & 0.01 & & \\
\hline \multirow{7}{*}{ Routine laboratory Data: } & $\mathrm{Hb} \% \quad \mathrm{gm} / \mathrm{dl}$ & -0.20 & 0.25 & -0.08 & 0.66 \\
\hline & $\begin{array}{l}\text { Platelet count thousands / } \\
\text { Cmm }\end{array}$ & -0.02 & 0.92 & 0.03 & 0.88 \\
\hline & WBC Count / c.mm. & 0.08 & 0.63 & -0.17 & 0.34 \\
\hline & PT Sec. & -0.08 & 0.66 & -0.21 & 0.23 \\
\hline & PTT Sec. & 0.01 & 0.94 & -0.05 & 0.77 \\
\hline & FBS mg/dl & 0.10 & 0.58 & -0.15 & 0.38 \\
\hline & P.P. bl. sugar : mg/dl & $-0.35(*)$ & 0.04 & -0.06 & 0.75 \\
\hline \multirow{8}{*}{ Routine hormonal data: } & FSH mi. u / ml & $-0.74(* *)$ & $<0.0001$ & $-0.92(* *)$ & $<0.0001$ \\
\hline & LH mi.u / ml & $0.48(* *)$ & 0.003 & $-0.84(* *)$ & $<0.0001$ \\
\hline & E2 pg./dl & -0.27 & 0.12 & $-0.95(* *)$ & $<0.0001$ \\
\hline & Prolactin $\mathrm{ng} / \mathrm{ml}$. & 0.15 & 0.39 & 0.18 & 0.30 \\
\hline & FT3 pg. / ml. & 0.15 & 0.40 & 0.06 & 0.73 \\
\hline & FT4 pg. / ml. & 0.13 & 0.45 & 0.31 & 0.07 \\
\hline & TSH $\mathrm{uIu} / \mathrm{ml}$ & 0.17 & 0.33 & 0.17 & 0.33 \\
\hline & progesterone $\mathrm{ng} / \mathrm{ml}$. & 0.14 & 0.42 & 0.22 & 0.20 \\
\hline \multirow{3}{*}{ Antiphospholipid antibodies. } & ACA $\quad$ IgG (GPL) & 0.14 & 0.43 & 0.01 & 0.97 \\
\hline & \begin{tabular}{|ll} 
ACA & IgU (MPL) \\
\end{tabular} & -0.24 & 0.17 & -0.06 & 0.75 \\
\hline & Lupus anticoagulant sec & 0.07 & 0.69 & -0.06 & 0.75 \\
\hline \multicolumn{2}{|l|}{ Antral Follicle Count } & $0.542(* *)$ & 0.001 & 0.643 & $<0.001$ \\
\hline
\end{tabular}

Table (3): Correlations between AMH and clinical data, routine laboratory data and routine hormonal data among patients. 


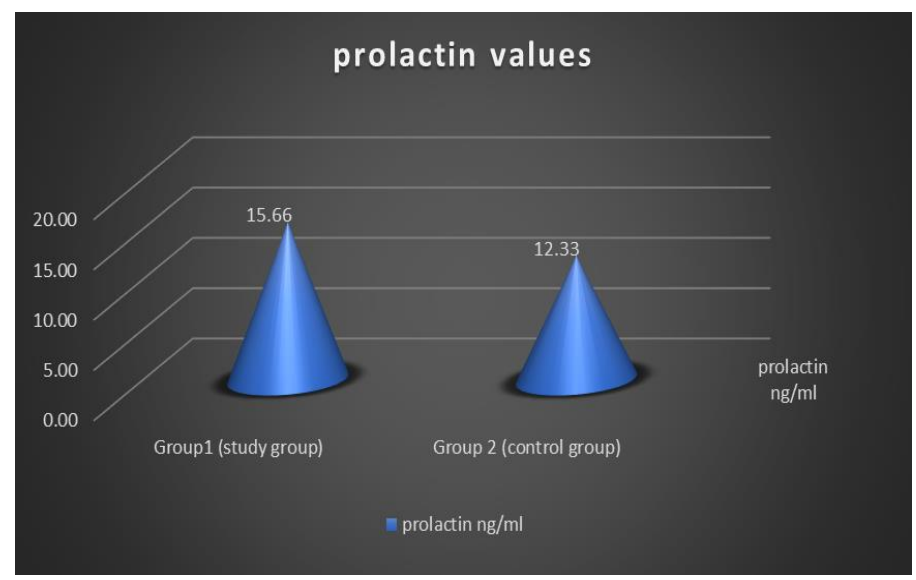

Figure (2): Comparison between patients and control group according to prolactin.

\section{DISCUSSION}

In the present study, the aim of our work was to try to evaluate Antral follicular count using trans-vaginal ultrasound and its relation to ovarian reserve in the patients with recurrent spontaneous abortions is defective or not by measuring serum levels of antiMüllerian hormone during ovarian follicular phase.

In comparison with other Several studies have revealed the same results which are Anti-Müllerian hormone and Antral follicular count have a positive correlation together, But the both have a negative correlation to the age, number of the +abortion, FSH \&LH.

There were 20 miscarriages among 28 pregnant women with highly elevated basal FSH levels, which a significantly increased miscarriage rate $(71 \%)$ is compared with a large control group with normal FSH levels. They included all sub-fertile women who visited their clinic; also women with irregular inclusion criteria were a basal FSH level, 10 IU/l and a proven ovulatory cycle. ${ }^{(10)}$.

Eighty women with recurrent pregnancy loss (RPL) was studied underwent routine work-up to exclude known associations of RPL. Serum FSH, LH and $\mathrm{E}_{2}$ levels were assessed on the 3rd day of the menstrual cycle. Following investigation, 58 women failed to reveal an identifiable cause and are therefore classified as unexplained RPL. Control group consisted of women in whom the cause of abortions was known such as uterine septum and parental chromosomal abnormalities. Mean age, gravidity, parity, presence of infertility, previous number of miscarriages, duration of marriage was similar in both groups. Day 3 serum levels of $\mathrm{FSH}, \mathrm{E}_{2}$ and FSH: LH ratios were compared in the two groups. They found that elevated FSH concentrations were equally distributed in the unexplained RPL and control groups. Both day $3 \mathrm{E}_{2}$ and FSH: LH ratio were elevated in the unexplained RPL group compared with the control group. The percentage of women with elevated FSH and/or $\mathrm{E}_{2}$ levels on day 3 was significantly higher in the unexplained RPL group than in controls. So they concluded that unexplained RPL may be associated with diminished ovarian reserve and should be considered in the workup of RPL ${ }^{(11)}$.

Twenty-eight women who miscarried were compared with 34 women who delivered a healthy baby after ICSI treatment. AFC proved to have predictive value for miscarriage, while female age, basal FSH and estradiol values did not ${ }^{(12)}$.

baseline concentrations of serum $\mathrm{AMH}$ and $\mathrm{FSH}$, and $\mathrm{AFC}$ were measured from 126 women undergoing IVF treatment. These data were then related to IVF outcomes. As expected, patients with lower serum AMH and antral follicle count (AFC) produced a significantly lower number of oocytes compared with patients with higher serum AMH/AFC. Fertilization rates in patients with lower serum AMH were significantly inferior compared with patients with higher serum $\mathrm{AMH}$, irrespective of whether IVF or intracytoplasmic sperm injection was used to achieve fertilization. These low AMH patients yielded fewer oocytes, had lower fertilization rates, generated fewer embryos, and had a 
higher incidence of miscarriage during fresh transfers, ultimately culminating in a halving of the pregnancy rate per $4 \mathrm{VF} *$ cycle compared with the high AMH group. So, Lekamge et al. demonstrated a significantly higher miscarriage rate amongst IVF treated women with low levels of AMH (5/17, 29.4\%) compared with women with high AMH levels $(6 / 36,16.7 \%)$. These results are of special interest since their study population was not at risk for severely decreased ovarian reserve. But the findings of Lekamge et al. have not yet been confirmed or rejected in other studies ${ }^{(13)}$.

Between January 1997 and December 2001, 2057 patients underwent 3401 consecutive IVF/ICSI cycles in which the basal level of FSH (days 2-4) was determined at an earlier cycle. Analysis, however, was only performed for a single cycle per patient. All cases were divided into four cohorts according to FSH levels. Higher levels of FSH were significantly associated with more cycle cancellation, a larger amount of gonadotrophin required to achieve follicular maturity, and a lower number of eggs collected, embryos available and embryos transferred. In all cases, however, there was no significant correlation between FSH levels and fertilization rate or miscarriage rate ${ }^{(14)}$.

The study was performed in women who were over 30 years of age without a history of subfertility, and pursuing a spontaneous pregnancy and was included 129 women without a history of subfertility pursuing a spontaneous pregnancy. Basal FSH concentrations were measured during three menstrual cycles. Urinary HCG levels were measured during menstruation for a maximum of six menstrual cycles, to detect early pregnancy loss. We estimated the effect of basal FSH concentrations on pregnancy outcome, taking into account possible confounders. They observed no significant effect of basal FSH concentrations on the incidence of early pregnancy loss or abortion of clinically recognized pregnancies and concluded that in a population of women without a history of subfertility, pursuing a spontaneous pregnancy; basal FSH concentrations are not related to the incidence of early pregnancy loss or abortions. The study therefore fails to confirm a relationship between signs of decreased ovarian reserve and aneuploid pregnancies ${ }^{(15)}$.
The follicular phase of women with recurrent pregnancy loss and a healthy control group was studied in a prospective casecontrol study in a tertiary care hospital of Sheffield, UK. Their populations were thirtyfour women with recurrent miscarriage and 10 women with no previous history of miscarriage regular menstrual cycles (control group) were recruited. The characteristics studied included Doppler assessment of blood flow to the follicle and the endometrium. Simultaneously, serum concentrations of biochemical markers such as anti-Müllerian hormone, inhibin $\mathrm{B}, \mathrm{FSH}, \mathrm{LH}$, and $\mathrm{P}$ were compared in the two groups. They were unable to detect significant differences in various biochemical and ultrasound measurements in the follicular phase between women with recurrent miscarriage and a control group; however, the expected correlation between ovarian and pituitary hormones, which was observed in the control group, was absent in women with recurrent miscarriage ${ }^{(9)}$.

a retrospective study within an IVF population was done and They analyzed 2,708 patients. Of these, 2,477 had normal basal FSH levels, and 231 had elevated basal FSH levels ( $>$ or $=13.03 \mathrm{IU} / \mathrm{L}$ ). Patients were segregated into various age groups. The miscarriage rate in the group with elevated basal FSH levels was $22.9 \%(11 / 48)$, similar to the controls with normal FSH $(19.3 \%, 226 / 1169)^{(16)}$.

A sub analysis was conducted within a prospective cohort study of 474 sub fertile ovulatory couples in two hospitals in Groningen, The Netherlands. The ovarian reserve tests (ORTs) performed was: antral follicle count (AFC), basal and stimulated levels of follicle-stimulating hormone (FSH) and inhibin $\mathrm{B}$, and the clomiphene citrate challenge test (CCCT). Women who achieved an ongoing pregnancy (n $1 / 4$ 233) were compared with women experiencing miscarriage (n 1/4 72) on the results of their ORTs and patient characteristics. In univariate analysis, the outcome of the ORTs did not differ between the groups. Logistic regression analysis including patient characteristics such as female age did not reveal an association between the ORT results and miscarriage either. They concluded that neither AFC, basal and stimulated levels of FSH and inhibin B, nor the CCCT have a statistically significant predictive value for miscarriage in sub fertile 
ovulatory women. They stated that they wanted to measure serum AMH for the study groups but unfortunately no serum was stored Since AMH was not such an acknowledged ORT at the start of the study in 1999. They said that it is a promising ORT ${ }^{(17)}$.

In correlation between various markers anti-Müllerian hormone was found to have negative correlation with age, number of abortions, FSH and LH. This is in agreement with Singer et al who concluded that serum $\mathrm{AMH}$ level, determined at random on any given day of a spontaneous menstrual cycle, is highly predictive of baseline FSH level obtained within 6 weeks e.g. an AMH level of $0.5 \mathrm{ng} / \mathrm{mL}$ is predictive of an FSH level of approximately $12 \mathrm{mIU} / \mathrm{mL}$. The presumed linkage in the relationship between baseline FSH and random AMH is that both hormones are indicators of ovarian reserve ${ }^{(18)}$.

Anti-Müllerian hormone in the control group was inversely related to basal estrogen levels, which may be explained on the basis that AMH inhibits the stimulating effects of FSH on small and pre-antral follicle growth, thereby preventing further growth and estrogen secretion. This negative correlation was lost in the patients group raising the possibility of subtle derangements of the feedback mechanism responsible for regulation of follicle development in this group of women. This is in agreement with work of Parakash et al as mentioned before ${ }^{(18)}$.

The finding that AMH was not different between both groups is surprising as a decreased quantitative ovarian reserve is considered to be a reflection of advanced ovarian ageing and ovarian ageing is clearly associated with an increased rate of fetal aneuploidy and miscarriage.

A possible explanation may be that AMH concentrations basically relate to the number of remaining oocytes and that their quantity is unrelated to their quality. Oocyte quality might predominantly be determined by biological damage accumulated over time and would thus not be related to the number of oocytes left, but mainly to female age ${ }^{(19)}$.

This hypothesis is compatible with recent publications demonstrating that female age does predict pregnancy chances, both spontaneous and after assisted conception, but ovarian reserve tests (ORTs) do not ${ }^{(4)}$.

Also, young women who respond poorly to ovarian stimulation during IVF treatment appear to have clearly better prospects than their older counterparts ${ }^{(20)}$.

Alternatively, it has been hypothesized that a biological relation between quantity and quality of oocytes does exist. The so-called production-line theory states that the germ cells produced earliest during fetal life are the least prone to non-disjunction. These oocytes are selected for ovulation first, leaving the oocytes of lesser quality for later years ${ }^{(19)}$.

The reason that ORTs do not predict miscarriage could hence be that these tests do not accurately reflect oocyte quantity. For instance, an elevated FSH level may indeed be due to a decreased number of follicles, but also to a range of other causes including the presence of heterophylic antibodies or FSHreceptor polymorphisms ${ }^{(21)}$.

Especially when the relation between oocyte quantity and quality is subtle, inaccurate estimation of quantitative ovarian reserve might obscure this relation. Finally, the etiology of miscarriage is known to be diverse. If any, ovarian reserve is only one of many contributing factors ${ }^{(21)}$.

Another possible explanation for the absence of a relation between ovarian reserve and the chance of miscarriage in our population is that the relation between oocyte quantity and quality does exist, but only at the very end of the reproductive period, when ovarian reserve is severely diminished, but the study excluded this group from the start (age between 20 and 40). Also, women with a severely decreased ovarian reserve were not likely to be included in the study population, since they often have irregular and an ovulatory cycle, which was an exclusion criterion in the study. In general, we cannot exclude the possibility that the relation between ovarian reserve and miscarriage does exist, but was not discovered in our study population, since differences between AMH levels may have been too small ${ }^{(21)}$.

Limitations of the study are that the size of study population might have been too small to detect a subtler relationship between ORTs and miscarriage. Also, the results of 
most ORTs may vary per cycle in the same woman, especially basal FSH ${ }^{(21,22)}$.

In this study, all ORTs were only performed once per participant. It is not known whether repeating these tests would enhance their predictive value for miscarriage in sub fertile populations. However, in a prospective study among fertile women, Van Montfrans et al. could not show a relation between repeatedly measured basal FSH and the chance of miscarriage ${ }^{(15)}$.

\section{CONCLUSION}

It seems from this small study that Anti-Müllerian hormone is the best indicator of ovarian reserve with a high sensitivity and specificity, so ovarian reserve of women with recurrent spontaneous abortions is not defective. Anti- Müllerian hormone and Antral follicular count don't play a great role in the cases of recurrent spontaneous abortions.

\section{REFERENCES}

1. Daya S (2004): Evidence-based management of recurrent miscarriage optimal diagnostic protocol. International Congress Series., 1266: 318:327.

2. American Society for Reproductive Medicine (2008): The American Society for Reproductive Medicine has recently revised its definitions of infertility and recurrent pregnancy loss. Fertil Steril., 90: S60.

3. Gruijters MJ, Visser JA, Durlinger AT, et al. (2003): Antimüllerian hormone and its role in ovarian function. Mol Cell Endocrinol., 15:85-90.

4. Broekmans FJ, Knauff EA, Valkenburg O et al. (2006): PCOS according to the Rotterdam consensus criteria: Change in prevalence among WHO-II anovulation and association with metabolic factors. BJOG., 113:1210-1217.

5. Cook CL, Siow Y, Taylor S et al. (2000): Serum müllerian inhibiting substance levels during normal menstrual cycles. Fertil Steril., 73: $859-61$
6. Fiçiciog C, Kutlu T, Elif Baglam et al. (2005): Early follicular anti müllerian hormone as an indicator of ovarian reserve. Semin Reprod Med., 23:248-256.

7. Fanchin R, Mendez Lozano DH, Frydman N et al. (2007): Anti$\mathrm{Mu}$ "llerian hormone concentrations in the follicular fluid of the preovulatory follicle are predictive of the implantation potential of the ensuing embryo obtained by in vitro fertilization. J. Clin. Endocrinol. Metab., 92:1796-1802.

8. Macklon NS and Fauser BC (2005): Ovarian reserve. Semin Reprod Med., 23:248-56.

9. Parakash A, Tin C, Laird S et al. (2006): Absence of follicular phase defect in women with recurrent miscarriage. Fertility Sterility, $85: 1784$

10. Levi AJ, Raynault MF, Bergh PA et al. (2001): Reproductive outcome in patients with diminished ovarian reserve. Fertil Steril., 76:666-669.

11. Gürbüz B, Yalti S, Ficicioglu C et al. (2004): High basal estradiol level and $\mathrm{FSH} / \mathrm{LH}$ ratio in unexplained recurrent pregnancy loss. Arch Gynecol Obstet., 270: 37-9.

12. Elter K, Kavak ZN, Gokaslan $H$ et al. (2005): Antral follicle assessment after down-regulation may be a useful tool for predicting pregnancy loss in in vitro fertilization pregnancies. Gynecol Endocrinol.,21:33-37.

13. Lekamge DN, Barry M, Kolo M et al. (2007): Anti-Müllerian hormone as a predictor of IVF outcome. Reprod Biomed Online, 14(5):602-10.

14. Abdalla H, Thum MY (2004): An elevated basal FSH reflects a quantitative rather than qualitative decline of the ovarian reserve. Hum Reprod., 19(4):893-8.

15. Van Montfrans JM, van Hooff MH, Huirne JA et al. (2004): Basal FSH concentrations as a marker of ovarian ageing are not related to pregnancy outcome in a general population of women over 30 years. Hum Reprod.,19(2):430-4

16. Luna M, Grunfeld L, Mukherjee T et al. (2007): Moderately elevated 
levels of basal follicle-stimulating hormone in young patients predict low ovarian response, but should not be used to disqualify patients from attempting in vitro fertilization. Fertil Steril., 87:782-7.

17. Haadsma ML, Groen H, Fidler $\mathrm{V}$ et al. (2009): The predictive value of ovarian reserve tests for miscarriage in a population of subfertile ovulatory women. Human Reproduction, 24:546-552.

18. Singer T, Barad DH, Weghofer A et al. (2009): Correlation of antimüllerian hormone and baseline follicle-stimulating hormone levels. Fertil Steril.,91: 2616-9.

19. Eichenlaub-Ritter U (1998): Genetics of oocyte ageing. Maturitas., 30:143-169.
20. Saldeen P, Kallen $K$ and Sundstrom $P$ (2007): The probability of successful IVF outcome after poor ovarian response. Acta Obstet Gynecol Scand., 86:457-461.

21. De Koning CH, McDonnell J, Themmen APN et al. (2008): The endocrine and follicular growth dynamics throughout the menstrual cycle in women with consistently or variably elevated early follicular phase FSH compared with controls. Hum Reprod., 23: 1416-1423.

22. Hendriks DJ, Broekmans FJ, Bancsi LF et al. (2005): Single and repeated $\mathrm{GnRH}$ agonist stimulation tests compared with basal markers of ovarian reserve in the prediction of outcome in IVF. J Assist Reprod Genet.,22:65-73. 